\title{
Application of Neural Networks and Radar Navigational Aids of Shore Area to Positioning
}

\author{
Tomasz Praczyk \\ Naval University, Śmidowicza 69, Gdynia, Poland \\ e-mail: T.Praczyk@amw.gdynia.pl
}

(Rec. 1 September 2005)

\begin{abstract}
The article presents the application of artificial intelligence techniques and data received from navigational radar i.e. information about distances to observed set of buoys to positioning on shore areas.
\end{abstract}

Key words: maritime navigation, neural networks

\section{INTRODUCTION}

In recent years, positioning in navigation has been dominated by satellite systems, especially by GPS. These systems are used at sea (maritime navigation), in the air (airplane navigation) as well as on the ground (land navigation). Basing solely on a satellite system carries, however, the risk of a sudden loss of navigational information in case of a sudden malfunction of such system, its power-down, inclusion of noise or coding data reaching the user. Therefore, there is necessity of being able to use an additional, autonomous (independent of external information sources) system that allows automatic position fixing.

In case of maritime navigation, one of the solutions is an application of information acquired from navigational radar. However, it does not involve position fixing in the classic way, i.e. with the help of crossing position lines determined for reference points of known positions. Presented approach assumes lack of navigator's knowledge about exact location of surrounding characteristic points. He cannot identify them (for various reasons), and therefore he is not able to determine position in the classic way. Presented idea is based rather on the image recognition methods. Comparative navigation is a prototype of it. It assumes that each position (near the coast) corresponds to characteristic radar image of a coastline. In short, comparative positioning consists in constructing a database of pattern radar images (from known positions) or a database of feature vectors determined for these radars images and then using all memorized information together with simple minimal distance algorithms during positioning process: the closest pattern image to the image recorded from navigational radar corresponds to the seeking position of a ship [7].
This approach has got two basic drawbacks. The first of them are difficulties with obtaining appropriate learning data for the system. The most natural, but at the same time the most expensive solution is registering real radar images from coast area of interest. In addition to huge costs of such undertaking, we will never be certain that data obtained in such way will represent everything that we can get on radar screen. In order for such data to become a reliable base on the ground of which we could fix position, they should be registered in various weather conditions, in different seasons, at night, in daytime and so on. All factors that could have an influence on radar image should be taken into account. Costs of such operation would be very high.

Another possibility of obtaining learning data is simulating them from paper or electronic chart. It is a cheap solution since it does not demand registration in real conditions. Unfortunately, it is not free from disadvantages. The first problem is difference between radar images and corresponding images generated from a chart. The differences concern remote elements of land as well as the same coastline. We can perform elimination of redundant fragments of land using, for example, invariant representation of image [3]. Unfortunately, determination of probable coastline posts not a small problem. In this case, it is necessary to have additional information about a nature of the coastline, for example, whether we deal with a beach or with a high cliff.

The second drawback of the method that uses pieces of land presented on radar image for positioning purposes are difficulties with location determination in areas with slight diversity of a coastline. In such areas, radar pictures even from distant positions could be very similar, making positioning impossible. The concept of using a contour of coastline obtained from navigational radar to positioning has therefore two essential shortcomings. The first are 
difficulties with obtaining reliable learning data, while the second is the fact, that we cannot apply this method everywhere we want.

That contributed to the idea of substituting the coastline, as a characteristic element for each position, for vector of distances to constant set of radar navigational aids, placed on considered area. Every ship is equipped with navigational radar, which enables determination of approximate distance and bearing to every object visible on radar screen - for example a buoy. Bearing and distance to some single object of known accurate position suffices to determine ship's location. The application of distance alone to fix position requires two points of known position. Therefore, if we only are able to attach to some object (or number of objects) visible on radar image its position, we can use it as a reference point and fix our position in relation to it by simple traditional radar navigation application. Unfortunately, identification of an object visible on radar image is not always possible even for an experienced navigator. For example, we can see on radar image some number of differentiable objects but we cannot assign to them identity i.e. their accurate positions. We can observe some number of potential reference objects but because of the fact that we do not know our position, sometimes even rough position (such situation should be seriously taken into account in case of navy ships), we cannot decide what objects are visible on radar image. It is necessary to remember that a situation observed on radar screen could be very similar in different regions of sea (it concerns buoys deployment visible on radar image). Moreover, information about location of every object could be additionally distorted because of errors generated by radar - bearing and distance fixed by radar are usually inaccurate. Difficulties in the reference objects identification could cause large number of misclassifications. Consequences of wrong decisions taken in a reference objects identification phase could be very serious. If the real position of reference object is strongly different than assumed position used during calculations then fixed position of our ship will be highly inaccurate. However, identification of potential reference objects is not the only problem connected with traditional navigational application. Assuming, however, that we are able to identify some objects, for example using information about their mutual location, another problem is that position fixed in traditional way, on the basis of noisy data could be also strongly inaccurate. Considering all these, we can risk a statement that the better solution then the traditional radar navigation could be the application of approximation tools. Maybe an approximator, in our case neural network, learned with the application of noisy data would be able to fix accurate ship's position.

It is assumed that every point at sea is characterized by the vector of distances (and possibly bearings) to buoys (which location is constant) that yield radar echo and are visible on radar image. In this case, we do not have to know an accurate position of every buoy, we do not have to know which buoy we can see on radar screen. Instead, we have an approximator which on the basis of distances to visible buoys is able to give us our approximate location at sea. In order to prepare such approximator it is necessary to have sufficiently large set of sample distances vectors coming from learning pattern points of known position. Very important issue, which should be taken into account during specific type of approximator choice, is the fact that it can encounter noisy data during work. Radar is not a perfect tool which can give us merely rough information about nearby objects. Distance and bearing to an object are usually noisy. For that reason, we decided that in the proposed conception, neural network would play the role of ship's position approximator.

Presented positioning approach has many advantages in comparison to the method based on the whole radar image or on features extracted from it. First and foremost, we can obtain learning data without necessity of expensive measurements at sea. In the previous case, in order to prepare the system we had to register appropriately large set of example pattern radar images and we had to do it at sea. In turn, distances vectors could be simulated without necessity of leaving a port. To do this, we have only to know location of every buoy considered as an element of the positioning system - we can read this information from a chart. Additionally, we have to fix a set of learning points, i.e. points which counterparts in the form of distances vectors will constitute learning examples applied during neural network learning. Equipped in such knowledge we can fix distances between every pattern point and a buoy. Distances vectors should contain only distances to buoys that are closer to the learning point than assumed radar observation range. Simply, radar has restricted field of vision and cannot see any object further then used radar observation range. Moreover, when generating distance vector for every learning pattern position we should remember about radar errors in fixing distance. Considering it consists only in adding some noise to exact distance value.

The next advantage of considered positioning method is the fact that its accessibility depends only on radar navigational aids presence in considered region. It is obvious that not every stretch of sea is covered with an appropriate set of radar aids, so in this case we also cannot apply this method everywhere we want. In this case, however, to enable positioning on a specific area it suffices only to adjust it by placing special buoys (beacons) on it. However, coastline is not modifiable.

Another great advantage of the presented method is its simplicity. Building the positioning system we do not demand any complicated and usually expensive tools - radar recorders and radar simulators. What is more, it is unnecessary to compress data that we use. These, which we apply, in comparison with radar images, contain slight amount of information - black and white $100 \times 100$-pixel picture 
includes 10000 units of information while presented conception uses vectors of distances maximally to 19 the closest buoys.

The range of the method i.e. size of sea area where we want to use a single copy of the system constitutes another problem. It is simply hard to obtain unique configuration of buoys deployment on large stretches of sea, what is the key factor determining accuracy of the system.

The article presents the same idea of radar navigational aids and neural networks application to positioning. We focused only on basic problems, including the possibility of suitably accurate positioning system based on existing radar navigational aids creation. Two important issues, that will be the essence of the future research, were omitted: the optimization of buoys deployment and using whole available information obtained from ARPA (radar) i.e. distances but also bearings to buoys.

\section{POSITIONING SYSTEM}

In order to check the presented conception of using radar navigational aids to positioning, research on the Gdańsk Bay area were carried out. Multilayered perceptron, learned with back-propagation algorithm and adaptive method of learning rate selection [4] and general regression neural network (GRNN) were used as a reasoning element.

The following structures of multilayered perceptron were used (input layer - hidden layers - output layer): 19-5-2, $19-10-2$, 19-20-2, 19-30-2, 19-40-2, 19-50-2, 19-100-2, $19-5-5-2, \quad 19-5-10-2, \quad 19-5-20-2, \quad 19-10-5-2, \quad 19-20-5-2$, $19-10-10-2,19-10-20-2,19-20-10-2$. The number of input neurons results from maximal number of buoys, that the system can "see" at given moment (we assume that maximally it could be 19 buoys). The Figure 19 is the number of real buoys located in the Gdańsk Bay area, originally considered as the element of the positioning system. The distances to observed buoys in the input vector are ordered from the distance to the most north buoy to the distance to the most south buoy. In case when we can see fewer then 19 buoys, appropriate network inputs are set to " 0 ". Two output neurons determine two coordinates necessary to fix position - latitude and longitude.

The number of input and output neurons of GRNN was the same as in the case of multilayered perceptron. The number of hidden (radial) neurons was dependant on applied training data net -400 or 1600 neurons (see next paragraph).

Real positioning systems, covering larger areas of a coast, in order to ensure adequate position accuracy could require great amount of learning data, which processing (during learning as well as during reasoning process) could take the system a lot of time. In case of memorizing solutions such as for example GRNN, application of great amount of learning data could cause delay in position fixing, what in turn leads to uselessness of navigational information acquired form the positioning network.

One of the solutions of the problem of great amount of learning information describing larger areas of the coast, is division of the whole set of data into groups or division of the considered body of water into sub-stretches. Obviously, division of learning data into groups corresponds to a certain division of a stretch into sub-stretches and likewise division of a stretch, results in division of data. Each group of data and each sub-stretch has separate positioning system. In case of the stretch division, in order to activate positioning subsystem for a given stretch, first it is necessary to decide in which area of sea our ship is located. This task could be realized by any identification system of the stretch (e.g. $\mathrm{kNN}$, another neural network). It would

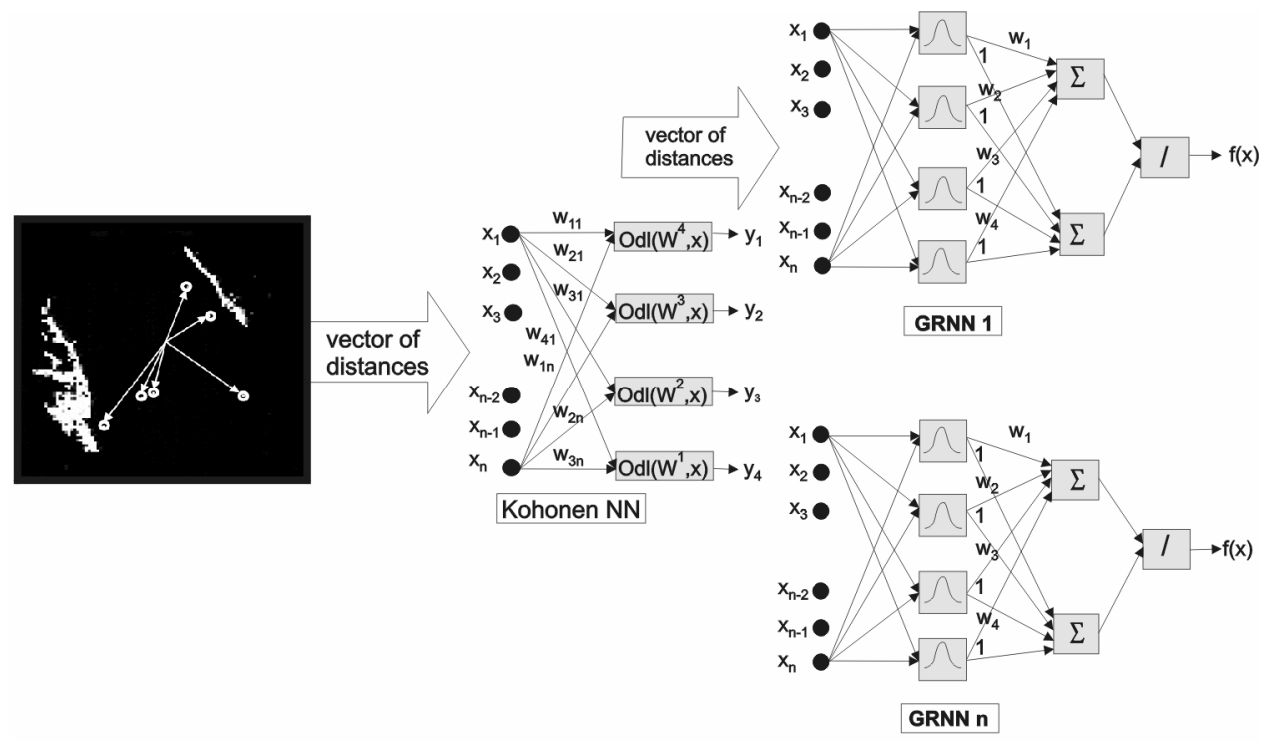

Fig. 1. The conception with a set of reasoning networks and with partitioning of learning dataset 
work on the basis of the vector of distances (distances and bearings) to whole set of visible buoys. At first, the vector would be created and then sub-stretch identification subsystem would decide where our ship is approximately located and then appropriate positioning subsystem would be activated in order to fix more accurate position within previously determined sub-stretch.

Data grouping is based on resemblance of data belonging to the same group. Separate group could for example constitute distances vectors with 16 visible buoys, next group with 15 visible buoys and so on. Another data division could be achieved through an application of any clusterization method e.g. self-organizing Kohonen neural network. In this case, decision which positioning subsystem should be activated would be taken on the basis of either quantity of buoys visible from a given place or similarity between registered vector and individual average distances vectors memorized in Kohonen network. The number of visible buoys would be counted and then, depending on the calculation result, appropriate positioning subsystem (corresponding for example to 15 visible buoys) would be activated. In case of Kohonen network application, created vector of distances would be compared to all neurons (average distances vectors) and positioning subsystem indicated by winner neuron (neuron the most similar to vector describing actual ship position) would be run. In case of Kohonen NN and GRNN application, the problem constitutes position determination on the edge of data cluster. GRNN is a radial network, thus position produced by this network for extreme areas of the cluster can be strongly inaccurate. To solve this problem GRNN is always built with application of data situated inside neighboring groups.

\section{DATASET}

The Gdańsk Bay region was the place where we decided to conduct experiments. The aids existing on the bay were used in the first stage of experiments. 19 of them, among all set of available buoys, were chosen to cover possibly the largest stretch of sea. After checking a possibility of positioning on the basis of existing buoys, in the second phase of the research the set of buoys were broadened by virtual buoys. They were placed in the locations of the highest position errors.

The reasoning, regardless of the stage of the research, took place on the basis of distances vectors to buoys visible in a given place and arranged in accordance with North South direction. The size of the vector was set to 19 distances (we were able to observe maximally 19 buoys). The research was carried out for the range of the radar observation $=12 \mathrm{Nm}$.

During experiments, radar errors in fixing distances to observed objects were taken into consideration. Each element of distance vector was a sum of exact distance from given position to definite navigational aid and the error from the range $\pm 0,02 *$ exact value.

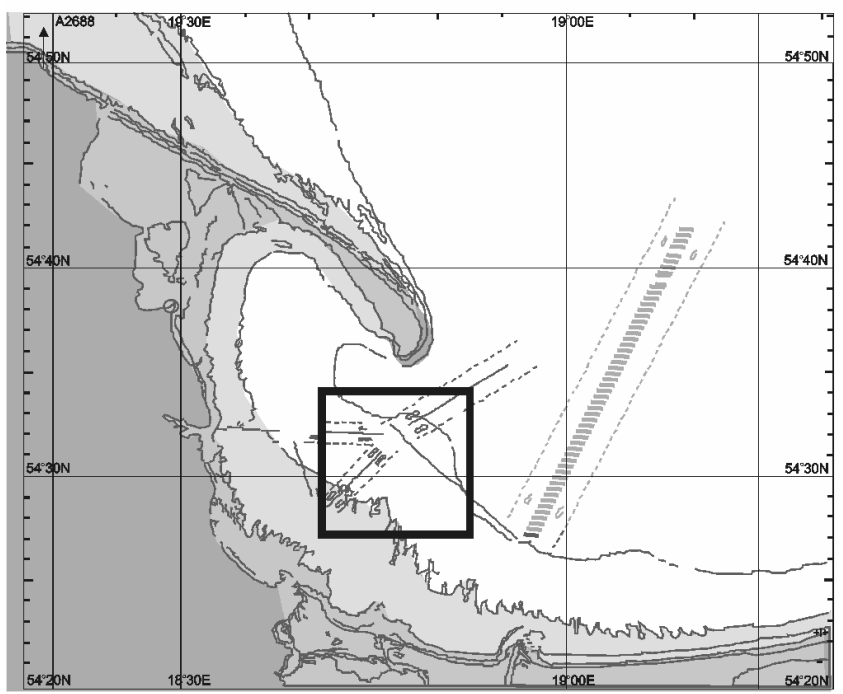

Fig. 2. Research area - marked by the black square

During learning two sets were applied. The first one included $400(20 \times 20$ learning points net $)$ learning points while the second one contained $1600(40 \times 40$ learning points net) points evenly placed on the research area.

The test data net arose through moving learning data in the east direction as well as in the north direction at a magnitude that was a half distance between neighboring learning points.

\section{EXPERIMENTAL RESULTS}

The research was divided into two stages. In the first stage possibility of positioning on the basis of an existing set of buoys was checked. Among whole set of buoys occurring on the Gdańsk Bay we selected 19 buoys. The second phase of the research consisted in checking influence of additional virtual buoys on accuracy of positioning system. New buoys were introduced in places of the highest errors of the positioning system.

In each stage, both solution with one neural network responsible for the whole considered area of sea and conception with division of learning data into groups were tested. Two methods of data division were applied. In case of the first method the criteria of division was the number of buoys visible from the given place i.e. one group presented distances vectors with 16 visible boys, another group with 15 visible buoys as far as to group with 7 visible buoys all in all 10 groups of data. The second method of division was self-organizing Kohonen NN. Neural gas algorithm was used to learn this network [4]. During research net- 
work consisting of 5 and 10 neurons was applied - 5 and 10 groups of data.

The learning distances vectors were used in order to build positioning subsystems as well as portioning Kohonen NN. Data from the test positions were used to measure performance of every examined solution. An average and maximum difference between pattern positions corresponding to test vectors and positions fixed by examined system for the given test distances vectors constituted accuracy measure of the system.

The research demonstrated that there is possibility of building coastal positioning system by means of neural network and characteristic points of a coast. The best outcomes were achieved by solution with the set of 10 GRNN and with partition of learning data with the aid of Kohonen neural network, for longer learning set. Maximum position error, which is the most important evaluation criterion of a positioning system, for this solution amounted to 0.0864 $\mathrm{Nm}$ (average error $-0.017 \mathrm{Nm}$ ). Considerably worse results were achieved by multilayered perceptron. The most precise positions, in this case, were obtained for the following structures of the network: 19-40-2 (400 learning points), 19-50-2 (1600 learning points). Worse performance of perceptron could have resulted from unmatching of every network structure to performed task. During learning of perceptron, back-propagation algorithm was used, which

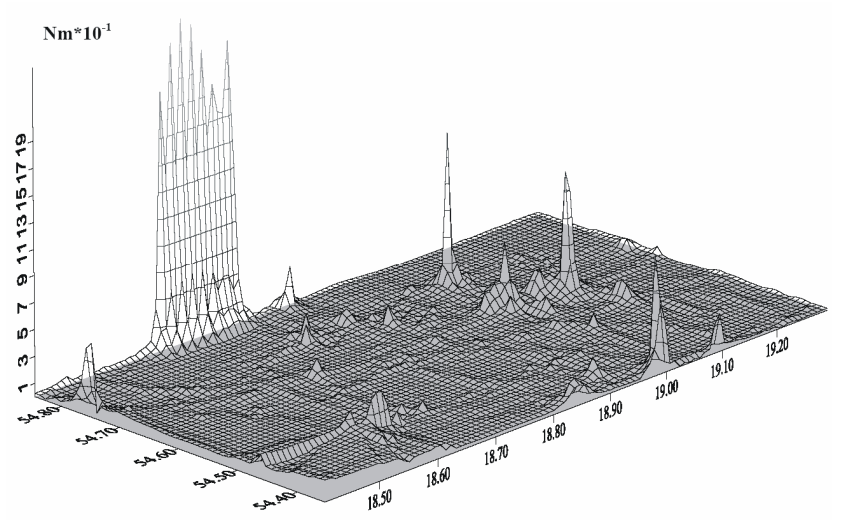

Fig. 3. Hypothetical position accuracy for the multilayered perceptron (19-10-2)

calculates optimal values of interneuron connections for network of fixed structure. The structure of each network was arbitrarily determined at the beginning of the learning process. No method allowing optimization of the network

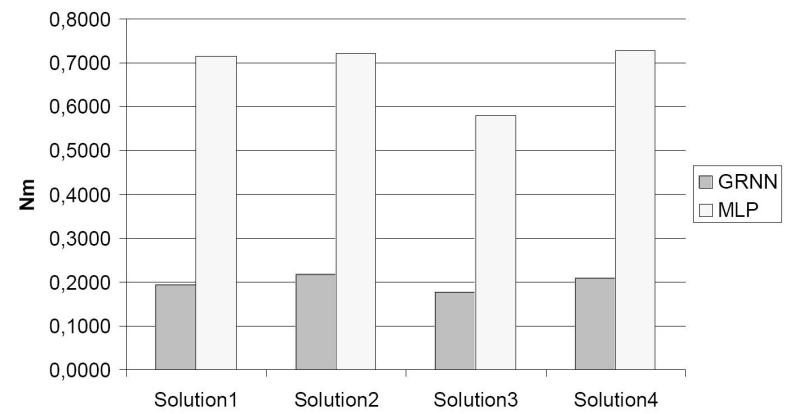

a)

Fig. 4. Maximum position error for different conceptions of the positioning system and for networks learned with the application of shorter (a) and longer (b) learning set (Solution1 - single copy of the network responsible for positioning on the whole considered area, Solution 2-10 positioning networks, learning dataset partition is based on differences in number of visible buoys, Solution 3-5 positioning networks, learning dataset partition is accomplished with the help of Kohonen network, Solution 4-10 positioning networks, learning dataset partition is accomplished with the help of Kohonen network)

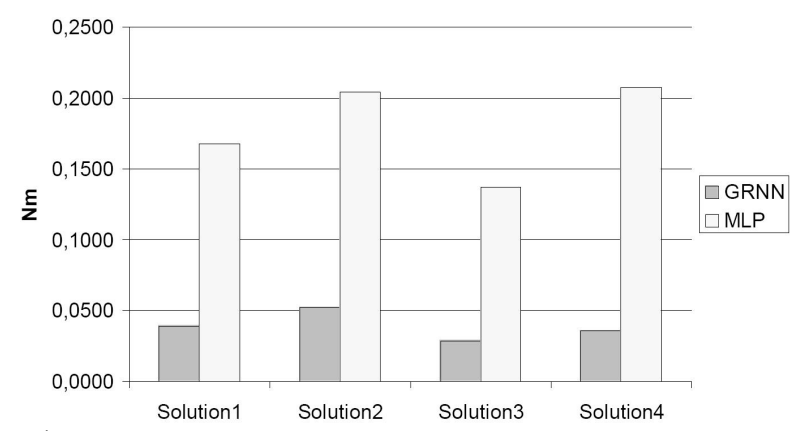

a)

Fig. 5. Average position error for networks learned on the strength of shorter (a) and longer (b) training set

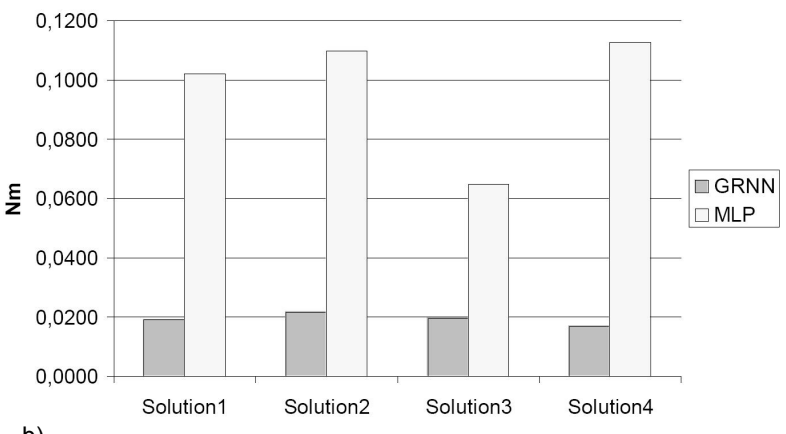

b)

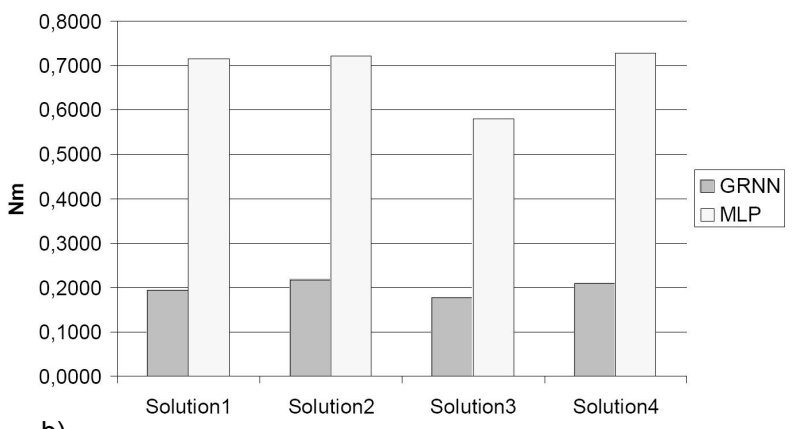

b) 
structure as well as synaptic weights was used (e.g. genetic algorithms). Willing to compare the proposed conception and traditional positioning we can use for this purpose maximum position error of every method. GRNN maximum position error is $0.0864 \mathrm{Nm}$, in turn perceptron's maximum position error amounts to $0.4142 \mathrm{Nm}$. The only thing that remains to be done is fixing the error for traditional radar navigation. We should consider two possible situations. First situation concerns the case of wrong classification of reference object and the second one assumes opposite situation i.e. correct identification of an object. In the first case position could be very inaccurate and the error may amount even to several miles. Regarding the second situation, let us consider the following case: we use only single object of known position to fix ship's location, bearing to the object is fixed without error (normally in navigation it is assumed that bearing error generated by radar amounts maximally to $2^{\circ}$ ), distance to the object is maximally distorted which means that it amounts to $0.02 *$ exact_value, and finally, we would like to fix our position on the basis of an object which is $12 \mathrm{Nm}$ away from our ship. In such situation the maximum error amounts to $0.02 * 12 \mathrm{Nm}=0.24 \mathrm{Nm}$. In order to improve position accuracy we can use of course more than one reference point. Thus, we can assume that maximum position error for traditional positioning is somewhat less than $0.24 \mathrm{Nm}$. Ultimately, comparing maximum position errors for traditional and proposed positioning method, we can state that their performance, particularly it concerns GRNN, is comparable.

Achieved outcomes demonstrate that regardless of positioning method, on the majority of considered area, position error is close to the average one. However, there still exist single points where position error significantly exceeds average value, coming sometimes even to 1 nautical mile. It is the error definitely too high for maritime positioning system.

A natural method of rare gross position errors elimination is learning data concentration in points where these errors appeared. An increase of the number of learning data entails unfortunately slowing down of the systems that work on the basis of memorizing solutions (e.g. GRNN) or causes considerable difficulties in creating valuable positioning system in case of parameterized methods (e.g. multilayered perceptron) application. Results of the procedure application consisting in supplementing training positions with additional 100 learning positions concentrated around 10 test points, where the highest position errors appeared, are depicted on the Fig. 6. Although learning set was expanded by slightly more than $6 \%$, outcomes are significantly better than results obtained with the application of original net of learning data.

Another accuracy improvement method is optimization of buoys deployment. It is necessary to adjust buoys de- ployment so that each two distant positions have strongly different equivalents in the distances vectors domain and simultaneously every two close positions correspond to nearby vectors.

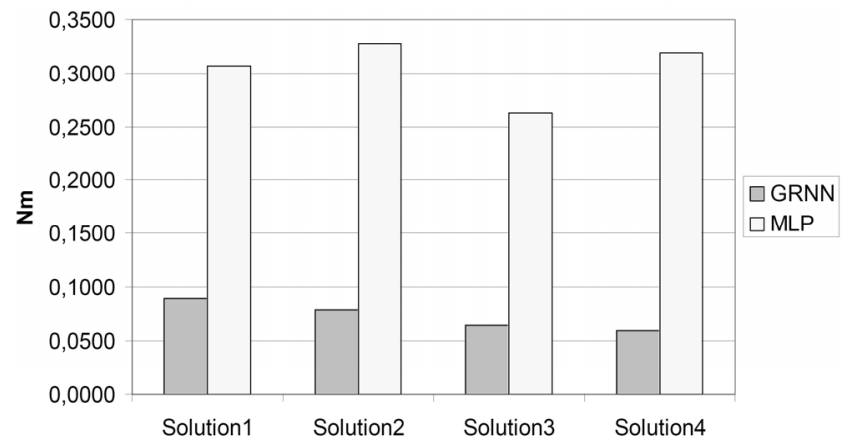

Fig. 6. Maximum position error for different conceptions of the positioning system and for networks learned with the application of broadened learning set -1700 learning points

The presented idea of the positioning system assumes that each position at sea area, where we want to use single copy of the system, is characterized by distances vector to visible and suitably arranged buoys. Information about bearings is completely omitted. Taking it into consideration would broaden knowledge, included inside vectors describing each position, making easier its fixing.

Building two separate, complementary positioning subsystems is also possible. The first that would work on the basis of distances to buoys and the second one would make use of bearings. Such solution would increase credibility of the entire system. Fixing approximately the same positions by two subsystems would increase navigator confidence in relation to both subsystems calculations.

Every ship is equipped with navigational instruments like log and compass, used in classical navigation, to fix movement direction and ship's velocity. Both information about state of a ship and additional information about ship's accurate initial position enables fixing approximate ship's position during a voyage. Unfortunately, only approximate position because log and compass, like radar, are not ideal tools and position fixed on the basis of values received from them deteriorates in the course of time. Therefore, log and compass can be successfully used only to eliminate gross position errors. Positions generated by our system that drastically differ from positions calculated with the use of log and compass should be rejected.

The reason why single gross position errors appear is large similarity between distances vectors corresponding to remote positions. Therefore, we decided to check whether increase of a number of buoys available on the considered sea area would change this situation. The problem was only to situate new buoys. We decided to use the simplest solution (we did not seek optimal locations for virtual buoys) 
consisting in introducing new virtual buoys into points where the highest position errors occurred. Hypothetical results of such solution are presented below.

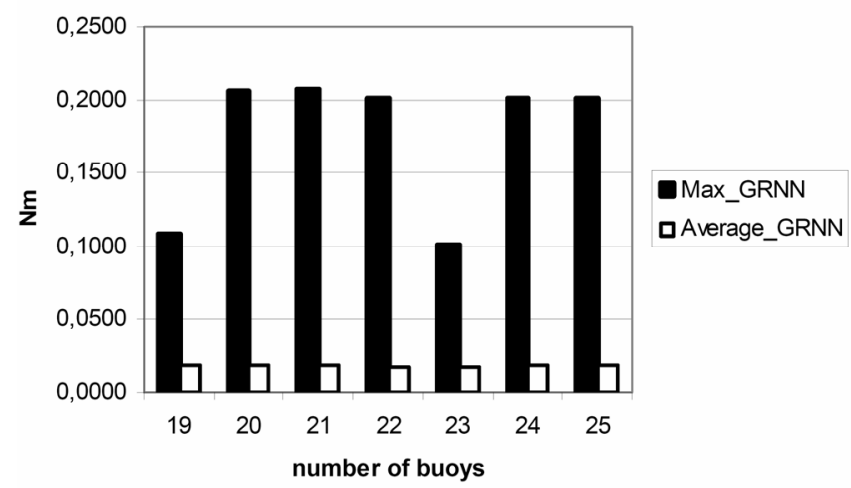

Fig. 7. The position error for GRNN and for longer learning set

Additional buoys did not improve results and, what is worse, in few cases they led to outcomes deterioration. The aim of broadening a set of buoys was divergence increase in a domain of distances vectors so as to facilitate distinguishing between individual regions of the considered sea area. Unfortunately, the approach that consisted in introducing additional virtual buoys into places of highest position errors appeared completely useless.

\section{CONCLUSIONS}

The article presents results of artificial intelligence technique (neural networks - GRNN, multilayered perceptron) and information about distances to the set of buoys application to positioning. The outcomes are very encouraging since they demonstrate that it is possible to built sufficiently accurate spare coastal navigational system with the application of presented tools.

The largest problem that appeared during research was sparse gross errors of position. On the majority of the considered sea area outcomes were satisfactory. However, single points occurred for which fixed position was considerably less accurate than for other test points. In the article the following methods of this undesirable effect elimination were presented: learning data concentration in places of the highest position errors occurrence, optimization of buoys deployment, correction of gross errors through navigational devices available on a ship application, and using all information that we are able to obtain from navigational radar i.e. bearings and distances, during building the system.

The next problem concerning the possibility of practical application of the proposed positioning conception is the fact that it uses set of buoys as characteristic objects. This causes that the method becomes useless in places without any radar navigational aids. However, research are conducted in order to generalize and to adapt this method also to regions deprived of navigational aids. In a future system, buoys placed on the considered region as well as automatically fixed characteristic points of land will play the role of reference points.

\section{References}

[1] B. Fritzke, A growing neural gas network learns topologies, Advances in Neural Information Processing Systems, MIT Press, Cambridge (1995).

[2] J. Korbicz, A. Obuchowicz and D. Uciński, Artificial neural networks, AOW PLJ, Warsaw (1994) (In Polish).

[3] G. Kuchariew, Processing and analysis of digital images, Szczecin Univ. of Technology, Szczecin (1999) (In Polish).

[4] S. Osowski, Neural networks in algorithmic sense, WNT, Warsaw (1996) (In Polish).

[5] T. Praczyk, The method of extraction of characteristic points from the radar image of the sea shore for the needs of positioning system, Annual of Navigation, no. 8, Gdynia (2004).

[6] T. Praczyk, Radar images compression for the need of a positioning coastal system and an assessment of this process, Annual of Navigation, no. 8, Gdynia (2004).

[7] A. Stateczny, Comparative navigation, Gdańsk Scientific Society, Gdańsk (2001) (In Polish).

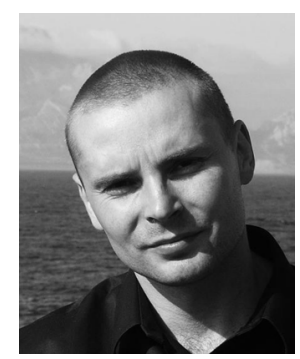

Tomasz Praczyk. Education: Military University of Technology, Warsaw - MSc (1996); Maritime University, Szczecin - PhD (2001). Activities: intelligent navigational systems, neural networks, genetic algorithms, neuroevolution, evolutionary reinforcement learning. 